\title{
On the Choice Between Such Simple Mechanisms as Individual Ownership and the First-come First-serve Rule When Renegotiation is Costly
}

\author{
Lando, Henrik
}

Document Version

Final published version

Publication date:

2004

\section{License}

CC BY-NC-ND

Citation for published version (APA):

Lando, H. (2004). On the Choice Between Such Simple Mechanisms as Individual Ownership and the Firstcome First-serve Rule When Renegotiation is Costly.

Link to publication in CBS Research Portal

\section{General rights}

Copyright and moral rights for the publications made accessible in the public portal are retained by the authors and/or other copyright owners and it is a condition of accessing publications that users recognise and abide by the legal requirements associated with these rights.

\section{Take down policy}

If you believe that this document breaches copyright please contact us (research.lib@cbs.dk) providing details, and we will remove access to the work immediately and investigate your claim. 


\section{Center for Law, Economics and Financial Institutions at CBS}

LEFIC WORKING PAPER 2004-01

On the Choice Between Such Simple Mechanisms as Individual Ownership and the First-come First-serve Rule When Renegotiation is Costly

Henrik Lando

www.cbs.dk/LEFIC 


\title{
On the Choice Between Such Simple Mechanisms as Individual Ownership and the First-come First-serve Rule When Renegotiation is Costly*
}

\author{
Henrik Lando ${ }^{\dagger}$ \\ Professor of Law and Economics \\ IVS and Lefic \\ Copenhagen Business School
}

February 13, 2004

\begin{abstract}
This article compares a set of often used simple contracts or mechanisms in terms of how well they allocate decision rights between two agents over time. A basic assumption is that agents incur a fixed cost each time they renegotiate. The contracts or mechanisms studied are: individual ownership and authority, the first-come first-serve rule, the
\end{abstract}

\footnotetext{
1 wish to thank Birger Wernerfelt, Oliver Hart, Nicolai Juul Foss and Kirsten Foss for helpful comments.

Corresponding address: Henrik Lando, Solbjergvej 3, DK-2000 Frederiksberg, Denmark; E-mail: hl.ivs@cbs.dk
} 
alternating rule and the sign-up rule. One trade-off that arises is the following: when usage of the asset is flexible in the sense that it does not matter in which period it occurs, agents may rely on obtaining the asset through arriving first at some point, while when an agent needs to time and plan the use of the asset, he or she may wish to hold stronger rights or to use the sign-up rule as a simple form of contracting. (JEL: D10, D23, L22); Keywords: Incomplete contracts, individual ownership, first-come first-serve rule, costly renegotiation. 


\section{Introduction}

When two agents share an asset or a resource over time, as when two households share a washing machine or when central and local management in an organization allocate decision rights between them, they often face a choice between mechanisms or contracts that are all simple ${ }^{1}$ and incomplete (i.e. less contingent than a Pareto-optimal contract). Presumably, the reason that only such mechanisms or contracts are realistic lies in the cost of elaborate contracting and in the fact that the mechanism or contract can be renegotiated as time passes, which diminishes the need for ex-ante contracting ${ }^{2}$. However, when it is costly to contract, it is also likely to be costly to recontract or renegotiate, which the agents should take into account when choosing between different kinds of simple mechanisms or contracts. This paper assumes that a fixed cost $\gamma$ is incurred each time renegotiation occurs, and analyzes which factors then determine the choice between simple contracts or mechanisms for allocating a good or resource over time.

The simple contracts or mechanisms are: the dictatorial right usually associated with individual ownership, the priority rule, the first-come first-serve rule, the alternating rule $^{3}$, and the sign-up rule. The dictatorial right grants all decision rights over an asset exclusively to one agent which implies that the others must ask permission of him or her before making decisions concerning the asset. The priority rule is a modified form of the dictatorial rule; it stipulates that one agent may use the asset whenever he or she desires, while the other may use the asset if and only if the agent with priority does not

\footnotetext{
${ }^{1}$ Simplicity of a mechanism will not be precisely defined in this paper, but it may be noted that the mechanisms referred to as simple do not require distinguishing different periods or contingencies and do not require memory on the part of the agents.

${ }^{2} \mathrm{On}$ the foundations of assuming incomplete contracts, see Hart and Moore (1999), Segal (1999) and Shavell (1998).

${ }^{3}$ The alternating rule will not be studied as much as the other mechanisms, but will be mentioned in some contexts.
} 
(actively) claim it. The difference between the dictatorial rule and the priority rule arises when the former agent does not express a desire to use the asset; the other agent may then use the asset if the priority rule - but not if the dictatorial rule - prevails. The first-come rule simply grants the decision right to the agent who arrives first to the asset ${ }^{4}$, while the alternating rule stipulates that the right to decide alternates through time. The sign-up rule allocates a given number of decision rights over the good to each agent who must then reserve the right to decide on the use of an asset for this number of future periods.

In the example of two households sharing a washing machine, all of the mechanisms are realistic possibilities, whereas in the example of the allocation of decision rights within a hierarchy, the main choice may be between the dictatorial rule and the priority rule. In a hierarchy, the dictatorial rule may be interpreted as the rule that local management may not implement (local) projects without the consent of central management while the priority rule may be interpreted as the rule that local management may do so unless central management has intervened ${ }^{5}$. However, the first-come rule may also be a possibility, implying that whoever has an idea first about how to use an asset (as modeled by Hart and Moore (1999)) may implement it.

Note also that in both of these examples the cost of renegotiation seems real. To change an allocation fixed by the simple mechanism, the two parties must get in contact, communicate and possibly bargain, all of which can be time-consuming activities.

Other simple contracts exist than those which will be analyzed in this paper. For example, a prevalent simple contract stipulates that one person may use the washing machine on all Mondays and Tuesdays, the other on all Wednesdays and Thursdays etc, and there exist simple contracts that employ the pay-peruse principle. However, since the purpose of this article is mainly to point to

\footnotetext{
${ }^{4}$ It is worth noting that arriving to the good may be interpreted as becoming ready to use the asset, e.g. because one gets an idea about how to use the asset, see Hart and Moore (1999).

${ }^{5}$ Thus, Hart and Moore (1999) associate the priority rule with hierarchy.
} 
a framework for analyzing the choice between simple contracts and to suggest some factors that are important in the choice of mechanism, no attempt is made to analyze the full set of realistic simple contracts.

The main factors determining the choice of mechanism may be discussed informally as follows ${ }^{6}$ : The first-come rule is flexible in the sense that if either of the two agents needs the asset at some point, perhaps unexpectedly, he or she may obtain the asset without bargaining if the other is not already using it. Thus, the first-come rule may economize on renegotiation costs. However, when an agent needs to plan the use of the asset, to time the use of it in order to fit it into a schedule, the agent may wish to have predictable access to the asset. Predictability may then be more important than flexibility. The agent for whom predictability is important may then wish to acquire stronger rights through the dictatorial rule, the priority rule or the sign-up rule. If both agents want predictability, the sign-up rule may be optimal, i.e. it may be optimal to enter into a simple kind of explicit contract. The sign-up rule may also eliminate uncertainty or risk: under the first-come rule, availability of the good may become uncertain if the other agent's utility is private information or if the other may often arrive first. An agent may then fear that his or her most important needs for the good will not be satisfied, especially when renegotiation costs are so high that the good cannot be obtained through renegotiation. The same risk is run by the non-owner in case of the dictatorial and the priority right while it may avoided through the simple explicit contract of the sign-up rule. This is the principle that contracting may be worth the cost when agents are risk averse and when contracting can eliminate or lower risk. However, if planning and the elimination of uncertainty cannot be attained in this way because needs are strongly stochastic, the sign-up rule loses some of its rationale and the first-come rule or -if needs are asymmetric- the dictatorial or the priority rule may be preferable.

In relation to existing literature, this paper follows the line of organizational research associated with, among others, Coase (1937), Simon (1951), Williamson (1985), Wernerfelt (1997), Baker, Gibbons and Murphy (2002a), (2002b), Hart and Moore (1999), Hart and Holmstrøm (2002), Levin and

\footnotetext{
${ }^{6}$ There are other factors, the analysis is also not exhaustive in this sense.
} 
Rayo(2003), and Simester and Knez (2002) in stressing the cost of contracting and recontracting (renegotiating).

Wernerfelt explains the choice between three mechanisms or game forms: the hierarchy-, the price list- and the negotiation-as-needed game form, in terms of how well they economize on communication costs while ensuring an efficient adaptation of sequences of acts. Thus, while the present and Wernerfelt's article do not analyze the exact same set of implicit contracts, they are closely related in that they both analyze the optimality of alternative implicit contracts when renegotiation is costly. As another difference, Wernerfelt does not apply the framework of arriving to the asset; he assumes, in the case of the hier archy, that there is a given string of actions, chosen initially by the employer, which an employee should carry out in the absence of adaptive instructions. Also, Wernerfelt assumes that there can be no communication when the game form is hierarchial, while this is only ruled out in this paper if $\gamma$ is high.

Hart and Moore (1999) define a hierarchy in terms of the priority right ${ }^{7}$ : In a centralized organization, the boss can implement his idea about how to use the asset (which can be interpreted as making a decision in some domaine) while subordinates can implement their idea if and only if the boss does not have an idea. As indicated above, 'getting an idea' in Hart and Moore's model can be interpreted within the framework of this paper, as 'arriving' to the asset. In this interpretation the present paper addresses why the priority right is often an efficient rule in hierarchies, and may extend the model by Hart and Moore by allowing bosses and subordinates to communicate and bargain about which idea should be implemented, which Hart and Moore rule out by assuming that $\gamma$ is prohibitively high.

Building on their prior work (2002a), Baker, Gibbons and Murphy (2002b) analyze how different kinds of strategic alliances between two firms may be thought of as different governance structures i.e. as different allocations of de-

\footnotetext{
${ }^{7} \mathrm{~A}$ working paper version of the present paper was written before the Hart and Moore paper (they make a reference to my working paper); in revising the working paper, I have extended the discussion of the role of planning (carrying out ideas) that Hart and Moore emphasize.
} 
cision rights over joint projects. The governance structure are: mergers (which they do not analyze), alliances (both firms have a veto right), acquisitions (one firm decides unilaterally, corresponding to the dictatorial right ), joint ventures (each of the two firms may carry out ideas, corresponding to the first-come rule) and divestitures (a third party owns the common project and is given autonomy). The authors assume that decision rights cannot be re-allocated through bargaining, and justify this by noting that one does not observe ownership flipping back and forth (see their footnote on page 12); however, they add that there exists some evidence that decision rights are renegotiated as alliances often become sales. In the context of the present paper, this evidence would suggest a high $\gamma$.

One central aspect of Baker, Gibbons and Murphy's framework is omitted from the present approach, namely the idea that the parties may act efficiently when they otherwise risk losing future rents from cooperation. This aspect is central also to the contribution by Levin and Rayo who derive a benefit in terms of economizing on relational capital of centralizing decision rights in the hands of one party ${ }^{8}$. In the present paper, it is assumed that relational capital is sufficient to induce the parties to respect the allocation of decision rights agreed upon ex-ante, but not sufficient to induce them to carry out the efficient decision in each period (e.g. to leave the asset to the other when that is efficient).

\section{The Model}

Two agents, $A$ and $B$, decide which mechanism to use for a given period of time, and are assumed to adhere to the mechanism that they have agreed on, i.e. the rule is assumed to be self-enforcing ${ }^{9}$. The period will consist of $n$ subperiods where only one of the two agents can use the given asset. In each

\footnotetext{
${ }^{8}$ The idea is, in short, that if a given future surplus provides an agent wiith an incentive to make one decision efficiently, the same surplus may also provide incentives for the same agent to make another decision efficiently.

${ }^{9}$ The implicit assumption is hence either that agents adhere to the rule because they have internalized the norm of not breaking agreements or that the game analyzed is part of a self-enforcing repeated game that for simplicity is not included.
} 
subperiod either $A$ or $B$ arrives first to the resource or asset ${ }^{10}$, and the rule then determines whether it is necessary for the agent to achieve the consent of the other agent before using the asset. If so, bargaining may take place and the one who has the decision-right (who is the first-comer in the case of a first-come rule) may require compensation to let the other use the asset. Also, the agent with the right may contact the other in order to rent out the asset for one subperiod.

Renegotiation is assumed to concern the allocation of the decision- right and not how the asset should be used to possibly satisfy the interests of both agents. The assumption is that one agent must carry out the decision, that he or she may know little about the needs of the other agent ${ }^{11}$, and that the other agent cannot easily communicate his or her needs to the decision maker. Under these circumstances it is realistic to assume that all which can be contracted for, both ex-ante and ex-post, are decision rights ${ }^{12}$.

Bargaining takes the form of take-it-or-leave-it offers by which the agents by exchanging two messages, an offer and a reply (acceptance or rejection), can realize the efficient allocation. The compensation should not necessarily be thought of as monetary; the idea is that they communicate to reach an agreement whereby the agent who needs the asset most obtains it, but must compensate the other in the form of a monetary payment or in the form of a future favor ${ }^{13}$. Each time renegotiation occurs, it is assumed to cost $\gamma$, reflecting not only the time spent bargaining but also the cost of getting in contact. Ideally, it would be preferable to provide microfoundations for this cost (e.g. in a model where agents may be more or less spatially separated and may reach each other through different media such as the telephone, e-mail or

\footnotetext{
${ }^{10}$ Naturally, we will consider the case where the probability of arriving first is affected by the person's need or by the person's rigth to use the asset.

${ }^{11}$ In the example of the washing machine, which clothes the other would prefer to wash.

${ }^{12}$ This assumption is also made by Baker, Gibbons and Murphy (2002b) in their static setting; they justify the assumption by reference to the action being unverifiable. Note, howeverm that they move on to suggest that actions and not only decision rights becomes contractible under relational contracting where the lack of verifiability plays a smaller role.

${ }^{13}$ Sometimes the future favor will not be agreed on but only expected which clearly saves on bargaining costs (but does not eliminate the cost of getting in touch and communicate).
} 
through approaching and talking to each other), but no such foundation will be provided in this paper.

$A^{\prime}$ 's utility over time can be written as $U_{A}(x)$ where $x=\left(x_{1}, x_{2} \ldots x_{n}\right)$, and $x_{i}=1$ if $A$ uses the good in subperiod $i$, while $x_{i}=0$ if $A$ does not. $B$ 's utility can similarly be written $U_{B}(y)$, where $y=\left(y_{1}, y_{2} \ldots y_{n}\right)$, and where $y_{i}$ is one or zero. As a special case, utilities over time may be additively time-separable. In this case, the utility of an allocation in any given period will be unaffected by what happens in other periods. Then $A$ 's utility in subperiod $i$ from using the asset will be termed $u_{A}^{i}$ while $B$ 's utility will be termed $u_{B}^{i}, i=1 \ldots n$. Utilities are assumed to be common knowledge, and non-stochastic ${ }^{14}$.

To fully define the priority rule, we must define what happens when $A$ has the priority right but $B$ arrives first and begins to use the asset before $A$ appears. $A$ then has the right to the asset but $B$ cannot be said to have violated $A$ 's right. In this situation, two polar cases will be analyzed; where $B$ 's use can be stopped at no cost in terms of production losses etc, and where it is simply not possible to interrupt $B^{\prime} s$ use of the asset. When interruption is possible, $A$ may contact $B$ to either tell him that she will take over the asset, or to extract a payment from $B$. In the former case, communication in itself will be assumed to cost $\gamma_{1}$, while when a payment is extracted the cost will be $\gamma$ as before, where $\gamma>\gamma_{1}$. If $A$ takes over the asset total utility will be $u_{A}^{i}-\gamma_{1}{ }^{15}$, while it will be $u_{B}^{i}-\gamma$ when $A$ extracts a payment from $B$. Furthermore, it can be assumed that when $A$ has priority or the dictatorial right and $B$ arrives first, $B$ will not attempt to extract a payment from $A$, since $A$ may then simply claim the asset. When interruption is not possible, and $B$ arrives first, it will be assumed that he will start using the asset when $A$ has the priority right but will abstain when $A$ has the dictatorial right.

\footnotetext{
${ }^{14}$ It seems worth exploring the consequences of assuming asymmetry of information, as this is both the more realistic case and the case which naturally leads to a cost of bargaining. However, introducing asymmetry of information complicates the analysis, and is not necessary for the purposes of this paper.

${ }^{15}$ Assuming also that $B$ neither suffers a loss from interruption nor has gained utility from using the asset before the interruption.
} 
Finally, it is assumed that the agents initially bargain in a Coasian fashion about the mechanism to be used, i.e. they choose the Pareto-efficient rule.

\section{Analysis}

The factors determining the choice of mechanism will be analyzed in the following three sections.

\subsection{On the role of relative needs}

The Pareto-efficient rule or mechanism depends on who needs the asset most as expressed in willingness to pay. The following assumptions constitute a benchmark for analyzing the importance of this factor:

(A1) In each period, both agents arrive to the good, and utilities are positive for both agents.

(A2) In all periods, the probability that one agent arrives first is independent of how much he or she values the good in that period.

(A3) For all subperiods $i$, utilities are independent of who obtained the good in subperiods prior to the $i$ 'th subperiod.

Given (A1)-(A3), the optimal strategies and their resulting total pay-offs can be calculated as follows: If $A$ has the dictatorial right $\left(A_{d}\right)$, and if she arrives first in period $i$, she will use the good if $u_{A}^{i} \geq u_{B}^{i}-\gamma$, otherwise she will at a cost $\gamma$ offer it to $B$ at price $u_{B}^{i}$. If $B$ arrives first, he will leave the asset if $u_{A}^{i} \geq u_{B}^{i}-\gamma$, otherwise he will ask $A$ for permission to borrow the asset for one subperiod at the rental price $u_{A}^{i}$. Hence, whether $A$ or $B$ arrives first, total utility will be $\max \left\{u_{A}^{i}, u_{B}^{i}-\gamma\right\}$. If $\mathrm{B}$ holds the dictatorial right $\left(B_{d}\right)$, total utility will by symmetry be $\max \left\{u_{B}^{i}, u_{A}^{i}-\gamma\right\}$. The set of periods for which $u_{B}^{i}-\gamma \geq u_{A}^{i}$ will be referred to as $\lambda$ while the set of periods for which $u_{A}^{i}>u_{B}^{i}-\gamma$ will be referred to as $\backslash \lambda$. 
The sum of expected utilities given $A_{d}$ is then given by:

$$
U_{\text {total }}\left(A_{d}\right)=\sum_{\lambda}\left(u_{B}^{i}-\gamma\right)+\sum_{\backslash \lambda} u_{A}^{i}
$$

The sum of expected utilities given $B_{d}$ is, by symmetry:

$$
U_{\text {total }}\left(B_{d}\right)=\sum_{w}\left(u_{A}^{i}-\gamma\right)+\sum_{\backslash w} u_{B}^{i}
$$

where the set of periods for which $u_{A}^{i}-\gamma \geq u_{B}^{i}$ is referred to as $w$ while the set of periods for which $u_{B}^{i}>u_{A}^{i}-\gamma$ is referred to as $\backslash w$. This brings out the following results concerning how utilities determine the allocation of the dictatorial right.

Proposition 1: If one agent's utilities is sufficiently high in all subperiods, that agent should have the dictatorial right.

Proof: This is obvious and follows directly from the fact that when e.g. A's utility in a given subperiod becomes sufficiently high compared to the utility of $B$, she should get the asset in that period, and this is achieved without bargaining if she has the decision right while it will only be achieved after bargaining when $B$ has the decision right (e.g. through arriving first). $Q E D$.

Proposition 2: Frequency of need counts more for the allocation of the dictatorial right than intensity of need.

Proof: This can be seen from the fact that very high needs (compared to $\gamma$ ) enter into the comparison only by their number and not by their magnitude, since the agent with a very high need will obtain the good regardless of who holds the decision-right, which only determines whether the agent will obtain the good with or without bargaining. $Q E D$.

Proposition 3: A change in $\gamma$ may change the optimal allocation of the dictatorial right. 
Proof: Consider the case where $A$ rarely but intensely needs the asset and relies on bargaining to obtain it she needs it, while $B$ needs the asset frequently but less intensely. If bargaining is not very expensive, it is likely to be optimal to give the dictatorial right to $B$. A will then obtain the good when her need arises, and $B$ can obtain the good in most of the periods without incurring the bargaining cost. If, on the other hand, bargaining becomes prohibitively expensive, $A$ will not obtain the good when her need arises, if $B$ has the dictatorial right. If $A$ 's need is sufficiently strong in those periods where she needs the asset, in comparison with the sum of $B$ 's needs, then $A$ should have the dictatorial right ${ }^{16}$. QED.

Proposition 4: Given assumptions (A1), (A2) and (A3), the first-come firstserve rule cannot be more efficient than the dictatorial right.

Proof: Given the first-come rule $(F C)$, if $A$ arrives first, this corresponds to the case where she holds the dictatorial right, making total utility in period $i$ equal to $\max \left\{u_{A}^{i}, u_{B}^{i}-\gamma\right\}$. If $B$ arrives first, total utility will by symmetry be $\max \left\{u_{B}^{i}, u_{A}^{i}-\gamma\right\}$ in period $i$. Total utility will therefore be

$$
.5 \max \left\{u_{A}^{i}, u_{B}^{i}-\gamma\right\}+.5 \max \left\{u_{B}^{i}, u_{A}^{i}-\gamma\right\}
$$

in period $i$ and hence:

$$
U_{\text {total }}(F C)=.5 U_{\text {total }}\left(A_{v}\right)+.5 U_{\text {total }}\left(B_{d}\right)
$$

since when $A$ arrives first, it is as if she holds the dictatorial right, and likewise for $B$. Since the sum of utilities is the average of the sum under $A_{v}$ and $B_{d}$, the claim follows. $Q E D$.

It will now be investigated under which circumstances the priority right prevails. Given the assumptions mentioned above about what happens when $A$ has priority but $B$ has begun using the asset, it will now be shown that the priority rule does not have a rationale when both agents need the asset in all periods:

\footnotetext{
${ }^{16}$ It may also be shown that the size of $\gamma$ may affect the relative desirability of individual and first-come when utilities are not always positive, but this is omitted.
} 
Proposition 5: Given (A1)-(A3), and if $u_{A}^{i}-\gamma_{1}$ is positive in all periods, the priority right cannot be more efficient than the dictatorial right.

Proof: Consider the choice between $A_{d}$ and the priority right $A_{p}$. Due to symmetry, the following analysis applies to the choice of giving the dictatorial or the priority right to $B$. In every period, $A$ will either want the good for herself or extract a payment from $B$ (since $u_{A}^{i}-\gamma_{1}$ is positive, $A$ will not want to simply leave the good to $B$ ). If the dictatorial right applies, $A$ will use the good herself when $u_{A}^{i}>u_{B}^{i}-\gamma$. If $A_{p}$ applies, $A$ will use the good herself when $u_{A}^{i}-\gamma_{1}>u_{B}^{i}-\gamma$. Thus, when $u_{A}^{i}>u_{B}^{i}-\gamma+\gamma_{1}, A$ will use the good herself under both rules; in this case the dictatorial right does better since it saves the cost $\gamma_{1}$ due to the fact that $B$ has not started using the asset, but has left it to $A$ who therefore does not need to communicate with $B$. If, on the other hand, $u_{A}^{i}<u_{B}^{i}-\gamma$, A will extract a payment from $B$ under both rules which means that welfare will be the same whether $A$ has one or the other right. What remains to be analyzed is the case where $u_{B}^{i}-\gamma<u_{A}^{i}<u_{B}^{i}-\gamma+\gamma_{1}$. Then $A$ will extract a payment under the priority rule but will use the asset herself under the dictatorial right. The comparison is hence between $u_{B}^{i}-\gamma$ and $u_{A}^{i}$ where the latter is higher, yielding the conclusion that the dictatorial right is uniformly better than the priority rule when usage can be stopped. When usage cannot be stopped, $B$ will use the asset when he arrives first since by (A1) his utility is positive and he cannot extract a payment from $A$ who has priority; thus, the priority rule excludes $B$ from selling to $A$ and hence induces a welfare loss compared with the situation where $B$ when arriving holds the dictatorial right. Thus, the total expected utility when $B$ arrives first is less when $A$ has priority than when $B$ has the dictatorial right. When $A$ arrives first, there is no difference between the situation where $A$ has the dictatorial or the priority right. Hence,

$$
U_{\text {total }}\left(A_{p}\right)<\frac{1}{2} A_{d}+\frac{1}{2} B_{d}
$$

from which it follows that the priority rule is either dominated by $A_{d}$ or by $B_{d}$ when usage cannot be stopped. QED

It will be shown below that the priority rule may well be optimal when one of the two agents does not always arrive to the asset. 


\subsection{The asset may be efficiently allocated without renegotiation}

Under some circumstances, the first-come rule and the priority right may avoid renegotiation while the dictatorial right requires it. The first instance of this can be seen when (A1) is relaxed; it will now be shown that if utility is zero for one of the agents in one subperiod, this in favors the first-come rule compared with the dictatorial right. Thus, if e.g. $A$ has zero utility and arrives at the good first, she may simply leave the good for the other agent to use ${ }^{17}$; this will be profitable if the cost of contacting $B$ is higher than the payment that can be extracted from $B$. In this case, the first-come rule may dominate the dictatorial right, since the first-come rule will costlessly allocate the asset to the agent who wants it the most, while the dictatorial right will not avoid bargaining costs in periods where the agent with zero utility holds the dictatorial right. Thus:

Proposition 6: Given assumptions (A2) and (A3), the first-come rule may dominate the dictatorial right when utilities are not always positive.

Proof: Since we only need to show that the first-come rule may dominate, an example is sufficient. Thus, imagine that in every period one agent has zero utility while the other's utility is lower than $\gamma$, and that the agent with zero utility alternates in each period. Then, given the dictatorial right, the good will be allocated inefficiently in every period where the one who has the dictatorial right has zero utility. If $A$ has the dictatorial right and arrives first, she will not contact $B$, and if $B$ arrives first, he will not contact $A$. Thus, in every second period, total utility will be zero. The first-come rule will, on the other hand, efficiently allocate the good in all periods without bargaining costs. Should the agent with zero utility arrive first, that agent will simply leave it for the other agent, since it will be too costly to get in touch with the other agent and establish an agreement ${ }^{18}$. QED.

\footnotetext{
${ }^{17} \mathrm{I}$ am grateful to Oliver Hart for pointing this out to me.

${ }^{18}$ Note that this example also brings out the essential and quite obvious strength of the alternating rule; if using the asset in one period means that utility in the next is small, the alternating rule is likely to be optimal.
} 
Another instance where the first-come rule dominates the dictatorial right occurs when (A2) is relaxed and when the probability of arriving first to the asset increases with need. Thus:

Proposition 7: If the probability of arriving first is positively influenced by the utility of the good in a given subperiod, the first-come rule may dominate the dictatorial right.

Proof: This can be seen by assuming that two kinds of subperiods exist: In the first kind, $A$ has no need for the asset, while $B$ does, and in the other kind, $B$ has no need for the asset, while $A$ does. If in the first kind of period, the probability is one that $A$ arrives first, and in the second kind of period, the probability is one that $B$ arrives first, then the first-come rule realizes the optimal allocation of the good without incurring bargaining costs, while e.g. $A_{d}$ incurs bargaining costs when $B$ arrives first to the good in those periods where $A$ has no need of the asset. $Q E D$.

More generally, the first-come rule will economize more on the need for bargaining the more an agent's effort to come first is increased with the utility the agent derives from the asset. However, since effort is costly, it may be excessive under the first-come rule, since a greater effort by one agent will impose an externality on the other ${ }^{19}$. The usefulness of the first-come rule will then depend on whether saved bargaining costs are more important than the cost of effort in arriving first.

The assumption of time-separable utility-functions, (A3), will now be relaxed to demonstrate a third factor that may obviate the need for renegotiation under the first-come rule. Two important aspects of utility functions can then be distinguished. First whether uses over time are substitutable and second whether utility of the good in period 2 is higher or lower for an agent when the agent has used the good in period 1, i.e. whether need is exhausted

\footnotetext{
${ }^{19}$ It is essentially the same reason why fishing effort will tend to be excessive. See Barzel (1974) for a study of the case where arriving first comes at the cost of spending time in a queue. Note also that under certain assumptions, a person who does not need an asset in a period may choose to exert zero effort, i.e. to drop out of the race.
} 
through use. To express whether need tomorrow is increased or decreased by today's use, the concept of super- and submodularity will be introduced ${ }^{20}$. If we restrict attention to two periods, supermodularity arises when $U_{A}(1,1)>$ $U_{A}(1,0)+U_{A}(0,1)^{21}$, while submodularity arises when $U_{A}(1,1)<U_{A}(1,0)+$ $U_{A}(0,1)$. The role of this distinction is made clear in the following.

First, note that in the case of two periods, when utility-functions for both $A$ and $B$ uses over time are sufficiently supermodular the dictatorial right is preferable to the first-come rule. To illustrate, if we let $U_{A}(1,1)=U_{A}(1,0)+$ $U_{A}(0,1)+c$ and $U_{B}(1,1)=U_{B}(1,0)+U_{B}(0,1)+c$, and $c$ is large enough, the same agent should of course use the asset in both periods, since this will yield a higher utility than both $U_{A}(1,0)+U_{B}(0,1)$ and $U_{B}(1,0)+U_{A}(0,1)$. $A$ should then have the right if $U_{A}(1,1)>U_{B}(1,1)$ and $B$ should hold it, if $U_{B}(1,1)>U_{A}(1,1)$. In either case, the efficient allocation will be obtained without bargaining given the dictatorial right.

Second, as will now be argued, when utility-functions are sufficiently submodular, the first-come rule dominates the dictatorial right when timing of the use of assets is not important: If $U_{A}(1,1)=U_{A}(1,0)+U_{A}(0,1)+c$ and $U_{B}(1,1)=U_{B}(1,0)+U_{B}(0,1)+c$, and $c$ is negative, intuitively the first-come rule may be thought to dominate when it is important that the good is used by one agent in one period and by the other agent in the other period, but this is not sufficient. When the sequence of use is important - when it is important e.g. that $A$ uses in the first period, and $B$ in the second - then the first-come rule will not save on bargaining costs. In this situation, when $B$ arrives first in the first period, bargaining is saved under the dictatorial right (but not under the first-come rule), while if $B$ arrives first in the second period, bargaining will be saved under the first-come rule (but not under the dictatorial right). So there is no net gain to the first-come rule in this situation. The advantage arises only when the sequence is not important. Then, when $A(B)$ arrives first to the good in the first period, she will use it in that period, and in the next period, $B(A)$ will obtain it, possibly through bargaining. When the sequence is not important, i.e. when the parties can afford to be flexible about

\footnotetext{
${ }^{20}$ See Milgrom-Roberts (1995) for the general definition of supermodularity.

${ }^{21} \mathrm{It}$ is assumed that $U_{A}(0,0)=0$
} 
who gets to use the asset in what period, bargaining in the first period can be avoided given the first-come rule. The conditions of sufficient submodularity and unimportance of timing can be stated formally as assumption 4 and 5, respectively:

Assumption 4: $U_{A}(1,1)-U_{A}(1,0)<U_{B}(0,1)-\gamma$ and $U_{B}(1,1)-U_{B}(1,0)<$ $U_{A}(0,1)-\gamma$

The first part means that when $A$ used the asset in the first period, it is worth shifting the dictatorial right in the second period to $B$, even at the cost of bargaining. The second part means that if $B$ used the asset in the first period, it is worth shifting it to $A$ in the second, even at the cost of bargaining.

Assumption 5: $U_{A}(1,0)=U_{A}(0,1)$ and $U_{B}(1,0)=U_{B}(0,1)$

This implies that both agents are completely indifferent about the period in which they get to use the asset. It is not a necessary assumption, but one that simplifies the analysis. Now:

Proposition 8: If $U_{A}(1,0), U_{A}(0,1), U_{B}(0,1), U_{B}(1,0)$ are all greater than $\gamma$ and Assumptions 4 and 5 above are fulfilled, the first-come rule dominates the dictatorial right.

The proof is in the appendix.

The point that the first-come rule may dominate when utility-functions are submodular can be seen clearly when the possibility that the agents become satiated (have no more need for the good) is explicitly introduced: If it is assumed that using the asset in one period yields utility $\sigma(>\gamma)$ which is the same for both agents, and that both agents are satiated in their demand for the asset when they have used the asset a certain number of times $\kappa$, total utility for $A$ is given by $\sum_{i=1}^{n} x_{i} \sigma$ when $\sum_{i=1}^{n} x_{i} \leq \kappa$, and by $\kappa \sigma$ when $\sum_{i=1}^{n} x_{i} \geq \kappa$. Similarly for $B$. Note the assumption that timing of uses is unimportant and 
that only the total number of uses counts. Then, the optimal strategy for each agent is to use the asset in each period in which the agent arrives first, until the point of satiation. In contrast, given the dictatorial right, bargaining costs will have to be incurred each time the non-holder wishes to use the asset. When the one who has the dictatorial right is satiated, the other will have to ask permission, and thus incur the cost $\gamma$ every time he wishes to use the asset. In this example, due to utilities of $A$ and $B$ being the same, the advantage of the first-come rule arises at the point where the one who has the dictatorial right is satiated (and not before, since the good goes to the one who has the dictatorial right in all prior periods without bargaining). Thus, when both $A$ 's and $B$ 's utilities are the same in every period, the first-come rule will dominate as soon as it is possible to satiate the one who has the dictatorial right. Thus:

Proposition 9: When satiation may occur as defined above, i.e when the number of periods $n$ is greater than $\kappa$, and probabilities of arriving are positive in each period for both agents, the first-come rule will dominate the dictatorial right

Proof: Let $n=\kappa+1$. Given the dictatorial right the one who has this right will obtain the asset in the first $\kappa$ periods. Then there will be bargaining in the last period. Given the first-come rule, there will be no bargaining in any period, and in every period, the asset will be efficiently allocated. Total utility given the first-come rule will be $(\kappa+1) \sigma$, while based on the dictatorial right it will be $(\kappa+1) \sigma-\gamma . Q E D$.

These insights lead to the conclusion that:

Proposition 10: The more utility-functions are super-modular and the more timing is important, the better is the dictatorial right in comparison with the first-come rule.

The results presented in this section have concerned the first-come rule in comparison with the dictatorial right. However, avoiding renegotiation is also important for the choice between the dictatorial and the priority right. Giving 
priority to $A$ is preferable to giving $A$ the dictatorial right when $A$ does not arrive. If $A$ does not arrive, and $A$ has the dictatorial right, $B$ will leave the asset. He will not approach $A$ who will not enter into a negotiation when she can simply claim the asset. When $A$ only has priority, $B$ will either use himself or approach $A$. Approaching $A$ yields nothing since $A$ may again claim the asset, instead of entering into bargaining. Thus, $B$ will use himself, and the utility gained relative to the situation under the dictatorial right is hence $\sum_{/ T}$ $u_{i}^{B}$ where $/ T$ is the set of periods where $A$ does not arrive. On the other hand, if $A$ does arrive, the question is who arrives first. If $A$ arrives first, there is no difference between the two rules. If $B$ arrives first, the difference is that $B$ may start using ${ }^{22}$ in which case $A$ will have to acquire the asset through contacting $B$ at the cost $\gamma_{1}$. In this case, when $A$ arrives and $B$ uses the asset, $A$ may instead extract a payment from $B$ in which case $u_{i}^{B}-\gamma$ is realized. This latter possibility is also present under the dictatorial rule when $A$ arrives and $B$ has left the asset for $A$ to use. Thus, under the priority rule the utility realized will be $\max \left\{u_{i}^{B}-\gamma, u_{i}^{A}-\gamma_{1}\right\}$ while it will be $\max \left\{u_{i}^{B}-\gamma, u_{i}^{A}\right\}$ under the dictatorial rule. Thus, when $u_{i}^{B}-\gamma>u_{i}^{A}>u_{i}^{A}-\gamma_{1}$, there is no difference between the utility realized under the two rules; if $u_{i}^{A}>u_{i}^{B}-\gamma>u_{i}^{A}-\gamma_{1}$, the loss under the priority rule will be $u_{i}^{A}-\left(u_{i}^{B}-\gamma\right)$ which is less than $\gamma_{1}$ by the inequality $u_{i}^{A}>u_{i}^{B}-\gamma>u_{i}^{A}-\gamma_{1}$; and finally if $u_{i}^{A}>u_{i}^{A}-\gamma_{1}>u_{i}^{B}-\gamma$ the loss under the priority rule compared with the dictatorial rule is $\gamma_{1}$. Thus, if $T^{*}$ is the set of periods where $A$ arrives and $u_{i}^{A}>u_{i}^{B}-\gamma$, the upper bound of the extra cost incurred by the priority rule is $\frac{1}{2} \sum_{T^{*}} \gamma_{1}=\frac{\gamma_{1}}{2}\left\|T^{*}\right\|$ where $\left\|T^{*}\right\|$ is the number of periods in $T^{*}$.

Thus:

Proposition 11: Given (A2) and (A3), when usage can be stopped at no (production) cost, priority to $A$ is preferable to the dictatorial right when $\frac{\gamma_{1}}{2}\left\|T^{*}\right\|<\sum_{/ T} u_{i}^{B}$, where $\left\|T^{*}\right\|$ is the number of periods where $A$ does arrive and $u_{i}^{A}>u_{i}^{B}-\gamma$, and $/ T$ is the set of periods where $A$ does not arrive.

\footnotetext{
${ }^{22}$ It is assumed that $B$ will start using even if he may be interrupted later by $A$.
} 
This means that the important question is how often $A$ will arrive and will need the asset enough to interrupt $B$ 's use instead of extracting a payment from $B$, and how often $A$ will not arrive at all where $B$ needs the asset. In other words, if $A$ needs the asset rarely but intensely (while $B$ frequently needs the asset), and $\gamma_{1}$ is not too high, the priority rule is preferable to the dictatorial.

\subsection{The role of uncertainty}

Uncertainty may concern the mechanism - one mechanism may create a more certain and predictable time-pattern of use than another- and uncertainty may also concern needs.

The uncertainty created by the mechanism is especially important when agents are risk-averse and when planning is important. When agents are riskaverse in the sense that welfare is increasing but at a decreasing rate with more access to the good, it becomes important that agents can be certain that they will not be too heavily rationed in their access to the asset. This implies that a highly risk-averse agent may insist on either the dictatorial or the priority right or on the sign-up rule despite it's set-up costs. This is the principle that contracting can be worth the cost when the elimination of uncertainty is important due to risk-aversion. Two more observations may be worth making: the alternating rule also eliminates the risk of heavy rationing, while the dictatorial and the priority right both implies that one of the agents is exposed to this risk.

Planning may involve making commitments to others (as a precondition for their commitments) and commitment may require predictability, i.e the absence of uncertainty. This aspect of planning and commitment connects to the analysis above: both supermodularity and timing may be associated with the planned use of the asset, for a plan often implies a timed, sequential scheme. The main implication is that when planning is important, uncertainty should be eliminated which it may be through the sign-up rule or the alternating rule. The dictatorial and the priority right only eliminates uncertainty for one agent. 
Uncertainty concerning needs affects the choice of mechanism in a different way from the uncertainty created by a mechanism. Thus, the advantage of predictable access vanishes when the need is itself unpredictable. If, for example, a households needs a washing machine when the kids soil their clothes and this happens stochastically over the week, the sign-up mechanism may not not be useful. Then other mechanisms such as the first-come rule (especially if the probability of arriving first depends on need) or the priority right to the household with small children, may be optimal. This reasoning is reminiscent of Simon's view concerning the advantage of authority over contract when there is a high degree of uncertainty. To model this aspect a stochastic framework is required where utility follows some stochastic process; this is left to future work.

\section{Applications to the theory of the firm and the theory of own- ership}

The relevance of the analysis to the theory of the boundary of the firm can be seen when the dictatorial and the priority right are interpreted as the right within an employment relationship to direct the use of an employee's time. The following example may illustrate: If the boss of law firm $A$ hires a lawyer $B$ to be part of $A^{\prime} s$ firm, it means that the boss can, within limits, tell $B$ to work on the case of a big client if the need for this work should arise, while if $B$ is an independent lawyer, the boss would have to persuade $B$ into working on the client's case, which may not be easy, if $B$ had planned to work on a case of one of his own clients. Bargaining might then occur, and may reach the optimal outcome, but this would come about at a cost that could well be high if the need is urgent. There would of course also be a risk of a hold-up which was not analyzed in this paper because it has been so extensively studied in the literature. However, due both to the risk of a hold up and to the cost of bargaining, $A$ 's ability to commit to the big client would be less if $B$ were not an employee. On the other hand, $B$ 's ability to commit to his own client is less when he is subject to $A^{\prime}$ s directions in which case any given commitment to $B$ 's own client may be interrupted by a direction given by $A$. The point is that the assumption of renegotiation or transaction costs is sufficient to generate a theory that incorporates both an advantage and a disadvantage of expanding 
the scope of a firm. In brief, when $B$ is independent, he does not have to incur the costs of bargaining with $A$ when he wishes to enter into a contract with his own client,- this is an advantage of independence. On the other hand, when if $B$ is an employee, $A$ can coordinate $B$ 's services in a predictable and bargaining-free way with the services of other employees or partners of the law firm without having to bargain with or persuade $B$ - this is an advantage of merging. Thus, when Coase (1937) pointed out the importance of haggling costs to an understanding of the scope of the firm, he could have argued that these were, in principle, sufficient to generate a theory of the boundary of the firm ${ }^{23}$.

The framework of the present article and some of the results derived above are also relevant to the theory of ownership. The first-come rule is often, e.g. in textbooks, associated with common or collective ownership while the dictatorial rule is associated with individual ownership. However, this is only partly justified. People owning assets together may use other mechanisms than the first-come rule, e.g. the sign-up rule ${ }^{24}$. Moreover, when an agent owns an asset, he or she may allow others to borrow it, and the rules for borrowing the asset may differ; the priority right may e.g. be used instead of the dictatorial. Thus, both individual and collective ownership may be supplemented by implicit contracts, and while the form of ownership matters to the determination of what happens when the implicit contract falls outside the self-enforcing range ${ }^{25}$, it should neither be assumed that common ownership is restricted to the first-come rule nor that individual ownership is restricted to the dictatorial or the priority right ${ }^{26}$. Note also that the comparisons made in this paper abstract from incentive issues, mainly the important fact that

\footnotetext{
${ }^{23}$ This is not to say, of course, that this factor alone can explain the observed boundaries of firms; arguably, a one-factor, general theory of the firm is probably not possible to construct.

${ }^{24}$ See e.g. Ostrom (1990). Also, clubs create mechanisms for sharing goods that are neither purely public nor purely private goods (see Adams and McCormick on the continuum of goods between private and public; for an overview of theory of clubs see e.g. Sandler and Tschirhardt (1980)).

${ }^{25}$ As stressed by Baker, Gibbons and Murphy (2002a) building on Grossman-Hart (1986) and Hart and Moore (1990).

${ }^{26}$ Nevertheless, a comparison of the dictatorial rights with the first-come rule remains relevant to the theory of ownership, since the creation of individual ownership in reality sometimes does replace the first-come rule of common (or no) ownership.
} 
individual ownership provides an incentive for creating and maintaining assets.

In general, it may be more fruitful to focus on the mechanisms themselves instead of one the categories of individual and collective ownership. Focusing on the concrete mechanism may even resolve, and throw further light on, disputes in the theory of ownership, as the following example illustrates: In his early contribution to the theory of ownership, Demsetz (1967) explains individual ownership in terms of the internalisation of externalities. As an example of the emergence of individual ownership, Demsetz mentions the rise of private property rights for land among North American tribes when fur trading increased. However, in a study of the actual (complex) structure of rights among aboriginal people, Bailey (1992) criticizes Demsetz's view ${ }^{27}$; Bailey emphasizes that when group hunting took place:

'a lineage group, a band, or village held common access, and the private property rights of "nuclear' families were irrelevant until the game was taken' (p. 191).

He concludes:

'I find that economic advantage sometimes rested clearly and positively with group exploitation of its entire territory'. (p. 195).

In terms of the mechanisms used, Bailey's observation is that Native Americans used the priority rule: Group hunting had priority over individual use, but the individual land owner could use his land as he wished, as long as it did not interfere with the hunting needs of the group. According to the analysis above, this was optimal since the group were likely to need access very much whenever it did need it, but did not need it very often. Whether one views

\footnotetext{
${ }^{27}$ As does Posner (1980) who argues that possessory rights ("usefruct") may be optimal in a primitive society.
} 
giving priority to the group (for hunting-purposes) as a form of collective or of individual ownership does not seem essential.

\section{Possible extensions}

The analysis of this paper can be extended in several directions, two of which shall now be mentioned.

First, questions on the enforcement of mechanisms may be addressed. Breach of the mechanism occurs; in the case of sharing of a washing machines e.g., this is known to be an area of anger and resentment between co-inhabitants. The anticipation of such outcomes may in turn affect the choice of mechanism. Thus, friends sometimes refrain from owning goods together for fear of losing the friendship in case of conflict. Furthermore, within the context of the model it may be asked why the implicit contract stipulating e.g. a first-come first -serve rule can be assumed to be adhered to while the parties cannot trust a mechanism that asks each agent to maximize total surplus in each period in which case all coordination problems could be solved ${ }^{28}$. But naturally an implicit contract that relies on truthful revelation of private information (need) is more fragile than an incomplete contract that relies on observables such as who arrived first to the asset ${ }^{29}$.

Second, there exist a set of norms and preferences that affect how the implicit contracts are played, especially within the private sphere, but which were not included explicitly in this paper. For example, the norm that favors are repaid in kind and rarely through monetary transfers among friends and relatives was mentioned but neither explained nor taken into account in the model. Also, when the model is applied to the private sphere of life it may be worth considering that some people attach a high value to altruism and communal living, and are hence less inclined to adopt individual property rights than people who value privacy and individual freedom, in part because it involves

\footnotetext{
${ }^{28}$ The question would then be which governance structure is better at supporting relational exchange as in Baker, Gibbons and Murphy (2002b).

${ }^{29}$ It was assumed that utilities were common knowledge but this assumption was made in order to simplify the analysis of renegotiation and not for realism.
} 
acceptance of individual utility maximization. Or that to many people a wellprotected sphere of privacy, i.e. the certainty that some decisions cannot be interfered with by others, is of importance in itself.

\section{Summary of results}

This paper has identified a set of commonly used simple mechanisms for allocating assets or resources over time and analyzed some of the main determinants of their efficiency within the framework of incomplete contracting and costly renegotiation. It has also suggested circumstances under which a simple form of ex-ante contracting is likely to be more efficient than the simple mechanisms that rely on ex-post renegotiation.

It may be worth summarizing some of the main results concerning the choice of mechanism.

One finding was that both the dictatorial rights and the first-come rule can be optimal. The dictatorial right, which enables the owner to make decisions concerning assets or goods without asking anybody else, is efficient compared with the first-come rule when arrival probabilities are exogenous, utility-functions are additively separable, and utilities are positive in all periods. However, when either one of the three assumptions is not fulfilled, the first-come rule may dominate the dictatorial right. Mainly, the first-come rule is likely to be preferable to the dictatorial right when

- the agent with the greatest need for the asset in a given subperiod is most likely to arrive first in the subperiod (without spending too much effort).

- timing is not very important (uses in different periods are close substitutes).

- uses over time are submodular, i.e. need is exhausted through use. 
Also, it was found that the priority right is preferable to the dictatorial when the one who has priority often does not need the asset but when in need, should get it. The the one who has priority may hence not be the agent who needs the asset most often, it is likely to be an agent who rarely arrives to the asset. The important thing is that when the agent arrives, the agent needs the asset more than the other, as is often the case in a hierarchy.

The sign-up rule may be efficient when uncertainty is costly due to riskaversion and when uncertainty of access makes it difficult to plan activities and make commitments. The sign-up rule permits both agents to plan, but only when utilities are themselves predictable (non-stochastic). When utilities are stochastic, the first-come rule may be preferable to 'contracting' (the sign-up rule), e.g. when probability of arriving first depends on need.

\section{Appendix}

Proof that the first-come rule is optimal when timing is unimportant and use should change hands:

Given the first-come rule, we can derive utilities as follows: Let us assume first that $A$ arrives first to the asset in the first period:

When $A$ arrives first, she can choose to use the asset herself. If she uses it herself in the first period, and also arrives first to the asset in the second period, she will sell to $B$ in the second period, since she can obtain the price $U_{B}(0,1)-\gamma$ which, by assumption 4 is greater than the gain in utility, $U_{A}(1,1)-U_{A}(1,0)$, from using the asset again in period 2. If $A$ uses the asset herself and $B$ arrives first in the second period, $B$ will use the asset. Hence, $A$ 's utility from using the asset will be $U_{A}(1,0)+.5\left(U_{B}(0,1)-\gamma\right)$. If $A$ does not use in the first period, she may either sell it to $B$ or leave the asset unused.

In the case where $A$ sells to $B$ in the first period, we will now derive the price that she can obtain from $B$. Denote the price by $p$. This price can be calculated as follows: If $B$ pays $p$ his total utility over the two periods will be 
$U_{B}(1,0)-p+.5\left(U_{A}(0,1)-\gamma\right)$. If $B$ does not buy the asset at price $p, A$ will use it rather than let it go to waste (since the utility of the asset is symmetric in the two periods there is no reason to let the asset go to waste), and $B$ 's utility will then be $.5 \times U_{B}(0,1)$, since he will derive no utility if $A$ arrives first in the next period and presents him with a take-it-or-leave it offer. Thus the price $p$ is given by:

$$
U_{B}(1,0)-p+.5\left(U_{A}(0,1)-\gamma\right)=.5 \times U_{B}(0,1)
$$

which yields: $p=U_{B}(1,0)+.5\left(U_{A}(0,1)-U_{B}(0,1)-\gamma\right)$. When $A$ obtains this price, his total utility from selling becomes: $p-\gamma+.5 \times U_{A}(0,1)$, where $\gamma$ is subtracted since there is a cost involved in selling at price $p$. She will hence sell if:

$$
p-\gamma+.5 \times U_{A}(0,1) \geq U_{A}(1,0)+.5\left(U_{B}(0,1)-\gamma\right)
$$

implying that

$U_{B}(1,0)+.5\left(U_{A}(0,1)-U_{B}(0,1)-\gamma\right)-\gamma+.5 \times U_{A}(0,1) \geq U_{A}(1,0)+.5\left(U_{B}(0,1)-\gamma\right)$

which again implies:

$$
U_{B}(1,0)-U_{B}(0,1)-\gamma \geq U_{A}(1,0)-U_{A}(0,1)
$$

By Assumption 5, this cannot be fulfilled, so $A$ will use the asset in the first period.

In the second period, $A$ will sell it to $B$ if $A$ arrives first, while $B$ will simply use the asset if $B$ arrives first. Thus when $A$ arrives first, we obtain the optimal allocation, and the expected bargaining cost is only $\gamma / 2$, since when $A$ has used to he asset in the first period, in the second period there will be bargaining 
only when $A$ arrives first again which occurs with probability $\frac{1}{2}$. So when $A$ arrives first, we can infer that the optimal allocation is attained at an expected bargaining cost of $\gamma / 2$. Since the case where $B$ arrives first is symmetric to the case where $A$ arrives first (the exact same analysis applies), we can conclude that the optimal allocation will be attained also in that case at an expected bargaining cost of $\gamma / 2$. Since $A$ and $B$ both arrive first with probability $\frac{1}{2}$, we can conclude that the optimal allocation is obtained given the first come rule at an expected bargaining cost of $\frac{1}{2} \times \frac{1}{2}+\frac{1}{2} \times \frac{1}{2}=\frac{1}{2}$.

Given the dictatorial right, the expected bargaining cost is higher. This follows from Assumption 4, according to which the asset must be used by both agents, but this means that the non-holder of priority must, in one of the two periods, either ask for permission to use the asset or by having the one who has priority sell it to him. Both ways involve bargaining which means that bargaining is certain to occur given the dictatorial right. This proves the proposition.

Note that it follows from this proof that when the optimal allocation is such that the asset must change hands and it is important that $A$ uses in the first period (in which case $B$ should use in the second), then there is no saving of transaction costs of using the first-come rule. In this case, if $B$ arrives first in the first period, there must be bargaining (expected cost: $\gamma / 2$ ), and likewise, if $A$ arrives first in the second period (expected cost: $\gamma / 2$ ). The total expected bargaining cost will then be $\gamma$, as when either $A$ or $B$ has the dictatorial right. QED.

\section{References}

[1] Adams R. and McCormick K.: Private Goods, Club Goods, And Public Goods As A Continuum, Review of Social Economy, 45, 1987. 
[2] Bailey M.: Approximate Optimality of Aboriginal Property Rights, Journal of Law and Economics, vol. XXXV, 1992.

[3] Baker G., Gibbons R. and Murphy K. J.: Relational contracts and the theory of the firm, The Quarterly Journal of Economics Vol. 117, Iss. 1; pg. 39, Feb 2002a.

[4] Baker G., Gibbons R. and Murphy K. J.: Relational Contracts in Strategic Alliances, working paper, August 2002b.

[5] Barzel, Yoram: A Theory of Rationing by Waiting, Journal of Law and Economics, XV11 (1), 1974.

[6] Coase R.H.: The Nature of The Firm, Economica 41937.

[7] Demsetz H: Toward a Theory of Property Rights, American Law Review, Papers and Proceedings (1967), p. 347-359.

[8] Grossman S. and Hart O.: The Costs and Benefits of Ownership: A Theory of Vertical and Lateral Integration, Journal of Political Economy, August 1986, 94 (4), pp. 691-719.

[9] Hart O. and Holmstrøm B.: A Theory of Firm Scope, Working paper, Harvard University, 2002.

[10] Hart O. and Moore J.: Property Rights and the Nature of the Firm, Journal of Political Economy 98 (6) 1990.

[11] Hart O. and Moore J.: On the Design of Hierarchies: Coordination versus Specialization, NBER Working Paper, 7388, October 1999.

[12] Hart O. and Moore J.: Foundations of Incomplete Contracts, Review of Economic Studies, January 1999, v. 66, iss. 1, pp. 115-38.

[13] Knez, M. and Simester, D.: Direct and Indirect Bargaining Costs and the Scope of the Firm, Journal of Business, v. 75, iss. 2, pp. 283-304, April 2002.

[14] Lando, H.: Communication Costs, Individual and Collective Ownership, mimeo, Copenhagen Business School, 1998. 
[15] Levin, J. Rayo, L.: Control Rights and Relational Contracts, working paper, Stanford and Chicago University, 2003, downloadable from http://gsbwww.uchicago.edu/research/workshops/wto/Documents/WTOspring2003.htm

[16] Milgrom, P. and Roberts, J.: Complementarities and fit: Strategy, structure, and organizational change in manufacturing, Journal of Accounting \& Economics; Amsterdam; Mar-May 1995.

[17] Ostrom,E.: Governing the commons: The evolution of institutions for collective action, Cambridge University Press, 1990, pages xviii, 280.

[18] Posner R.: A Theory of Primitive Society with Special Reference to Law, Journal of Law and Economics, 23 (1980).

[19] Sandler T. and Tschirhardt, J.: The Economic Theory of Clubs: An Evaluative Survey, Journal of Economic Literature, Vol 23, December 1980.

[20] Segal, I.: Complexity and Renegotiation: A Foundation for Incomplete Contracts, Review of Economic Studies, vol 66, no. 1, 1999.

[21] Shavell, S.: Contracts, New Palgrave Dictionary of Law and Economics, (ed. Peter Newman), 1998, downloadable from http://papers.ssrn.com.

[22] Simon, H.: A Formal Theory of the Employment Relationship, Econometrica, 19, 1951.

[23] Wernerfelt B.: On the Nature and Scope of the Firm: An Adjustment Cost Theory, Journal of Business, Autumn, 1997.

[24] Williamson O.: The Economic Institutions Of Capitalism, New York Free Press (1985). 